\title{
Interaction between HCMV pUL83 and human AIM2 disrupts the activation of the AIM2 inflammasome
}

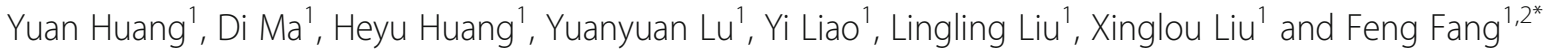

\begin{abstract}
Background: AIM2, a cytosolic DNA sensor, plays an important role during infection caused by pathogens with double-stranded DNA; however, its role in human cytomegalovirus (HCMV) infection remains unclear. Previously, we showed an increase in AIM2 protein levels during the early stage of HCMV infection and a decrease $24 \mathrm{~h}$ post infection. Because HCMV has developed a variety of strategies to evade host immunity, we speculated that this decline might be attributed to a viral immune escape mechanism. The tegument protein pUL83 is an important immune evasion protein and several studies have reported that pUL83 binds to specific cellular proteins, such as AIM2-like receptor IFI16, to affect their functions. To determine whether pUL83 contributes to the variation in AIM2 levels during HCMV infection, we investigated the pUL83/AIM2 interaction and its impact on the AIM2 inflammasome activation.

Methods: We constructed plasmids expressing recombinant pUL83 and AIM2 proteins for two-hybrid and chemiluminescence assays. Using co-immunoprecipitation and immunofluorescent co-localization, we confirmed the interaction of pUL83/AIM2 in THP-1-derived macrophages infected with HCMV AD169 strain. Furthermore, by investigating the expression and cleavage of inflammasome-associated proteins in recombinant HEK293T cells expressing AIM2, apoptosis-associated speck-like protein (ASC), pro-caspase-1 and pro-lL-1 $\beta$, we evaluated the effect of pUL83 on the AIM2 inflammasome.

Results: An interaction between pUL83 and AIM2 was detected in macrophages infected with HCMV as well as in transfected HEK293T cells. Moreover, transfection of the pUL83 expression vector into recombinant HEK293T cells stimulated by poly(dA:dT) resulted in reduced expression and activation of AIM2 inflammasomeassociated proteins, compared with the absence of pUL83.

Conclusions: Our data indicate that pUL83 interacts with AIM2 in the cytoplasm during the early stages of HCMV infection. The pUL83/AIM2 interaction deregulates the activation of AIM2 inflammasome. These findings reveal a new strategy of immune evasion developed by HCMV, which may facilitate latent infection.
\end{abstract}

Keywords: HCMV, pUL83, AIM2 inflammasome, Immune evasion

\section{Background}

Human cytomegalovirus (HCMV) is one of the most ubiquitous pathogens in the world. In immunocompetent individuals, HCMV infections usually progress to lifelong persistent latency after a short-term lytic infection, unaffected by the host immune system. HCMV has evolved multiple strategies to circumvent the innate and adaptive

\footnotetext{
* Correspondence: ffang56@163.com

'Department of Pediatrics, Tongji Hospital, Tongji Medical College, Huazhong University of Science and Technology, Wuhan 430030, China

${ }^{2}$ Teaching and research office of pediatrics, Tongji hospital, Jiefang Road No. 1095, Qiaokou District, Wuhan 430030, China
}

immune responses to establish such a long period of coexistence in the host [1-3]. The immune evasion is ascribed to the 230-kbp viral genome and enormous proteome [4].

pUL83 (also termed pp65) accounts for 15\% of total virion protein [5] and is the most abundant tegument protein. It plays a role during cell entry and in the transcription of immediate-early (IE1 and IE2) genes [6, 7]. In addition to these roles in viral physiology, pUL83 is involved in immune evasion, which is pivotal during HCMV infection. For instance, pUL83 phosphorylates IE proteins to prevent immunological recognition of the virus $[8,9]$. Interferon (IFN) levels in 
fibroblasts infected by the $\mathrm{HCMV} \triangle \mathrm{pp} 65$ strain, a mutant virus lacking the UL83 open reading frame (ORF), are higher than those in cells infected with wild-type virus. In contrast, overexpression of pUL83 partially blocks the IFN response, indicating that pUL83 is irredundant in suppressing the cellular IFN response to HCMV infection [10]. Moreover, pUL83 directly and specifically binds natural killer (NK)-activating receptor NKp30 to suppress the activation of NK cells [11].

Absent in melanoma 2 (AIM2) protein contains a C-terminal hematopoietic IFN-inducible nuclear (HIN) domain, which recognizes double-stranded (ds) DNA; and an N-terminal pyrin domain, which binds to apoptosisassociated speck-like protein (ASC) and subsequently recruits pro-caspase- 1 for its auto-cleavage and proinflammatory cytokine maturation [12-17]. The AIM2 inflammasome is indispensable during certain infections [18]. Although its role in the immune response to HCMV remains unclear, multiple studies have provided indirect evidence for the possibility that AIM2 can recognize HCMV, as follows. (i) The HIN domain of AIM2 recognizes dsDNA through electrostatic interactions, irrespective of the DNA sequence and GC content, but in a length-dependent manner $[17,19]$. (ii) Aim2, a murine homologue of AIM2, plays an important role in mouse cytomegalovirus (MCMV) infection [18]. (iii) Several researchers reported that $\mathrm{HCMV}$ infection induces the secretion of inflammatory cytokines such as interleukin (IL)- $1 \beta$ in serum of renal transplant recipients who developed a primary HCMV infection and IL-18 produced by HCMV-infected gingival fibroblasts [20, 21]. Even though the presence of Z-DNA binding protein 1 (ZBP1) was sufficient to enhance HCMV-stimulated transcription and secretion of IFN- $\beta$, its role in the release of IL- $1 \beta$ and IL18 remains unconfirmed [22]. This suggests the existence of other immune pathways that activate these two cytokines during HCMV infection. Furthermore, Cristea et al. reported that HCMV pUL83 hijacks IFI16 to activate the major immediate early promoter (MIEP) through binding to the pyrin domain of IFI16 [7, 23]. Considering that AIM2 is in the same protein family as IFI16 and has a pyrin domain, we hypothesized that pUL83 is involved in the immune evasion of AIM2 inflammasome in a proteinprotein interaction-dependent manner. Verification of this hypothesis comprises the aim of this study. We analyzed the interaction between pUL83 and AIM2 in recombinant HEK293T cells using two-hybrid and chemiluminescence assays. We also used co-immunoprecipitation and immunofluorescent co-localization experiments to study the interaction in HCMV-infected THP-1-derived macrophages. Furthermore, we evaluated the impact of pUL83 on AIM2 inflammasome activation in recombinant HEK293T cells expressing AIM2, ASC, pro-caspase-1, and pro-IL1ß.

\section{Methods}

Cells and virus

MRC-5 and HEK293T cells were sustained in Dulbecco's modified Eagle medium (DMEM) (Gibco) contained $10 \%$ newborn calf serum (Gibco). THP-1 cells were cultured in RPMI 1640 medium contained 10\% fetal bovine serum (Gibco). HCMV AD169 strain was propagated in MRC-5 cells and stored in liquid nitrogen.

\section{Competent cells and plasmid vectors}

Stellar Competent Cells (Clontech) were stored at $-80{ }^{\circ} \mathrm{C}$. Luria-Bertani (LB) medium (yeast extract, peptone, $\mathrm{NaCl}$ ), with or without agar, was proceeded autoclaving before adding ampicillin $(50 \mu \mathrm{g} / \mathrm{ml})$ or kanamycin $(30 \mu \mathrm{g} / \mathrm{ml})$, and stored at $4{ }^{\circ} \mathrm{C}$. pM GAL4-BD Cloning Vector (pM, $3.5 \mathrm{kbp}$ ), encoding the DNA binding domain (BD) of GAL4, pVP16 AD Cloning Vector (pVP16, $3.3 \mathrm{kbp}$ ), encoding activating domain (AD) of GAL4, and pG5SEAP Reporter Vector (pG5SEAP), containing secreted alkaline phosphatase (SEAP) gene with an upstream activating sequence (UAS) were contained in Matchmaker ${ }^{\mathrm{rm}}$ Mammalian Assay Kit (cat. 630305) purchased from Clontech, as well as pM3-VP16 Positive Control Vector (pM3-VP16), pM-53, pVP16-T and pVP16-CP. All vectors contain ampicillin resistance gene. pDsRed2-N1, containing kanamycin resistance gene, was used to recombine AIM2 inflammasome proteins expression vectors.

\section{Reagents}

Restriction endonuclease (EcoRI, SalIand BamHI), PrimeScript $^{\mathrm{tm}}$ II 1st strand cDNA Synthesis Kit and

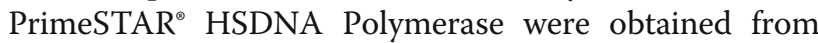
Takara. Gel Extraction Kit, Plasmid Extraction Kit and Endo-free plasmid kit were from Omega. In-Fusion ${ }^{\circ} \mathrm{HD}$ Cloning Kit (Clontech, cat. 639648) was used for inserting desired genes into vectors. CalPhos ${ }^{\mathrm{TM}}$ Mammalian Transfection Kit (Clontech, cat. 631312) was for transfecting reconstructed plasmids into mammalian cells, and Great EscAPe SEAP Chemiluminescence Detection Kit (Clontech, Cat. 631701) was bought for assaying SEAP. Phorbol myristate acetate (PMA) (Sigma, USA) was applied for THP-1 cell differentiation. Protein A/G bead was from Thermo Fisher Scientific, pUL83 antibody (Abcam, cat. ab6503), AIM2 antibody (CST, cat. D5X7K), ASC (Santa Cruz, cat. sc-30153), caspase-1 antibody (Santa Cruz, cat. sc-515), IL-1 $\beta$ antibody (Biovision, cat. 5128) and fluorescent tagged second antibodies were used at recommended concentrations. 4', 6diamidino-2-phenylindole (DAPI) was used at $2 \mu \mathrm{g} / \mathrm{mL}$.

\section{Primer design}

UL83 ORF (1686 bp, GenBank: KJ743149.1), AIM2 mRNA (1032 bp, NCBI Reference Sequence: NM_004833.1), ASC mRNA (588 bp, GenBank: AB023416.2), Caspase-1 mRNA 
(1209 bp, NCBI Reference Sequence: NM_012762.2) and IL-1 $\beta$ mRNA (810 bp, NCBI Reference Sequence: NM_000576.2) were picked as templates to design primers using Clontech online prime design tool for In-Fusion. According to the user manual of In-Fusion clone, the 5' end of each primer was deliberately added 15 bases that are homologous to 15 bases at one end of the vector (italics) and restriction enzyme sites (bold italics) were fully retained (Table 1).

\section{Construction of recombinants plasmids Linearization and purification of vectors}

$\mathrm{pM}$ and pDsRed2-N1 were digested by EcoRI and Sall overnight at $37{ }^{\circ} \mathrm{C}$, while pVP16 was digested by EcoRI and BamHI overnight at $30^{\circ} \mathrm{C}$. Agarose gel electrophoresis (AGE) isolated fragments were subsequently purified by Gel Extraction Kit according to the user guide.

\section{Reverse-transcription polymerase chain reaction (RT-PCR) and purification of fragments}

HCMV AD169 infected THP-1-derived macrophages were collected to extract total RNA and synthesize cDNA, which used as templates to amplify desired genes using indicated primers.

\section{In-Fusion cloning}

ORFs of UL83 and AIM2 were respectively inserted into linearized $\mathrm{pM}$ and $\mathrm{pVP} 16$ vectors; ORFs of ASC, caspase-1 and IL-1 $\beta$ were inserted into linearized pDsRed2 vectors separately according to In-Fusion ${ }^{\circledR} \mathrm{HD}$ Cloning Kit user manual. Reaction mixtures were then

Table 1 Primers for genes of interest

\begin{tabular}{|c|c|c|}
\hline \multirow[t]{2}{*}{ UL83 } & forward & $\begin{array}{l}\text { 5'-TGTATCGCCGGAATTCTCAACCTCGGT } \\
\text { GCTIITG-3' }\end{array}$ \\
\hline & reverse & $\begin{array}{l}\text { 5'-CTGCAGACGCGTCGACATGGAGTCGC } \\
\text { GCGGTCGC-3' }\end{array}$ \\
\hline \multirow[t]{2}{*}{ AlM2 } & forward & $\begin{array}{l}\text { 5'-GTACGGTGGGGAATTCGGAGGCTGATC } \\
\text { CCAAAGTTGT-3' }\end{array}$ \\
\hline & reverse & $\begin{array}{l}\text { 5'-ACGCGTCGACGGATCCTGCTGCTTAGAC } \\
\text { CAGTTGGC-3' }\end{array}$ \\
\hline \multirow[t]{2}{*}{ ASC } & forward & $\begin{array}{l}\text { 5'-CTCAAGCTTCGAATTCATGGGGCGCGC } \\
\text { GCGCGAC-3' }\end{array}$ \\
\hline & reverse & $\begin{array}{l}\text { 5'-CCGCGGTACCGTCGACTCAGCTCCGCT } \\
\text { CCAGGTC-3' }\end{array}$ \\
\hline \multirow[t]{2}{*}{ caspase-1 } & forward & $\begin{array}{l}\text { 5'-CTCAAGCTTCGAATTCATGGCCGACAA } \\
\text { GGTCCTG-3' }\end{array}$ \\
\hline & reverse & $\begin{array}{l}\text { 5'-CCGCGGTACCGTCGACTTAATGTCCTG } \\
\text { GGAAGAGG-3' }\end{array}$ \\
\hline \multirow[t]{2}{*}{$I L-1 \beta$} & forward & $\begin{array}{l}\text { 5'-CTCAAGCTTCGAATTCATGGCAGAAGT } \\
\text { ACCTGAGC-3' }\end{array}$ \\
\hline & reverse & $\begin{array}{l}\text { 5'-CCGCGGTACCGTCGACTTAGGAAGACA } \\
\text { CAAATTGCAT-3' }\end{array}$ \\
\hline
\end{tabular}

Italics: 15 bases homologous to one end of vector. Bold italics: restriction enzyme sites transformed to competent cells for incubation on resistant medium plates.

\section{The confirmation of recombinants}

Monoclone was proliferated in LB liquid medium before plasmid extraction. Recombinants were roughly identified by double enzyme digestion and PCR. pM-UL83 was digested with EcoRI and Sall at $37{ }^{\circ} \mathrm{C}$ and pVPAIM2 was digested with EcoRI and BamHI at $30{ }^{\circ} \mathrm{C}$. Undigested recombinant plasmids were used as templates for the amplification of desired genes. Digested and PCR products were subjected to AGE. Sequencing confirmation was then applied. Plasmids were extracted with Endo-free plasmid kit and transfected into HEK293T cells for $72 \mathrm{~h}$ and the expression of recombinants were detected by SDS-PAGE (12\%).

\section{Two-hybrid and chemiluminescence assay}

HEK293T cells were seeded into $10-\mathrm{cm}$ petri dishes and incubated for $24 \mathrm{~h}$. Then the medium was replaced by fresh DMEM complete medium and incubated for another $2 \mathrm{~h}$ or more until the cells achieve $70 \%$ confluence. Using the calcium phosphate transfection method, plasmids were transfected into HEK293T cells. $8 \mathrm{~h}$ later, calcium phosphate-containing medium was exchanged by DMEM complete medium and cells were incubated for $72 \mathrm{~h}$. Supernatant of each dish was centrifuged to discard cell debris and then subjected to SEAP detection by chemiluminescence at $405 \mathrm{~nm}$. Statistical data were analyzed by $T$-test.

\section{Co-immunoprecipitation}

Cells were harvested and lysed with cold protein lysis buffer (50 mM HEPES, pH 7.4, $250 \mathrm{mM} \mathrm{NaCl,} \mathrm{0.1 \%}$ NP-40, 2 mM EDTA, 10\% glycerol, protease inhibitors cocktail) for $30 \mathrm{~min}$. Then centrifuged at $12000 \mathrm{rpm}$ for $10 \mathrm{~min}$ at $4{ }^{\circ} \mathrm{C}$. Supernatant of cell lysates were transferred into new tubes and mixed with primary antibodies and incubated at $4{ }^{\circ} \mathrm{C}$ with gentle agitation overnight. Then protein A/G beads was added to capture antigenantibody complex, which subsequently proceeded heat denaturing and immunoblotting.

\section{Immunoblotting}

Cells lysates were prepared as mentioned above. Heat denatured cell lysates were then subjected to SDS-PAGE and transferred to PVDF membranes. The membranes were blocked with 5\% skim milk and incubated with primary antibodies overnight at $4{ }^{\circ} \mathrm{C}$, and subsequently incubated with horseradish peroxidase (HRP)-conjugated secondary antibodies before processing exposure. 


\section{Immunofluorescent}

Cells were washed twice with cold phosphate buffer saline (PBS), and fixed with $4 \%$ paraformaldehyde for $10 \mathrm{~min}$. Then appropriate amount of $0.3 \%$ TritonX-100 was added. Normal non-immune serum was used to block non-specific epitopes. Cells were incubate with specific primary antibodies overnight at $4{ }^{\circ} \mathrm{C}$, and subsequently incubated with fluorescent labelling secondary antibodies before observing with fluorescence microscope.

\section{Statistical analysis}

The means of triplicate samples were compared using $T$-test statistical method with GraphPad Prism software (GraphPad Software, USA). A $P$ value of $<0.01$ was considered as statistically significant.

\section{Results}

Plasmids for expression of recombinant pUL83 and AIM2 proteins

MRC-5 cells were infected with HCMV AD169 strain for $2 \mathrm{~d}$, until pUL83 was highly expressed [24]. The cells were then collected and UL83 and AIM2 genes were amplified by RT-PCR. The genes were used as templates in subsequent in-fusion cloning.

The pM GAL4-BD cloning vector was used to construct the pM-UL83 vector, where the UL83 ORF was inserted into the multiple cloning site (MCS) (Fig. 1a). The AIM2 ORF was cloned into the pVP16 AD cloning vector to fuse AIM2 with AD (Fig. 1b). The recombinant plasmids pM-UL83 and pVP-AIM2 were first verified by restriction endonuclease cleavage and PCR (Fig. 1c). Further nucleotide sequencing revealed $100 \%$ sequence identity with the UL83 and AIM2 genes. Good expression of the recombinant pUL83 and AIM2 proteins were observed in HEK293T cells (Fig. 1d).

\section{Recombinant pUL83 and AIM2 proteins interact with each other in mammalian cells}

We detected an increase in AIM2 protein levels in THP1 -derived macrophages $3 \mathrm{~h}$ post HCMV infection, which gradually increased up to $12 \mathrm{~h}$. However, the level was lower at $24 \mathrm{~h}$ than at $12 \mathrm{~h}$ for unknown reasons (unpublished data). To investigate whether the attenuation of the AIM2 inflammasome was linked to HCMV pUL83, we first determined the interaction between pUL83 and AIM2 using two-hybrid system. The main principle of the two-hybrid system is that $\mathrm{BD}$ and $\mathrm{AD}$ will act together as a transcriptional activator if they are tethered in space, even if they belong to separate proteins $[25,26]$. Accordingly, an interaction between pUL83 and AIM2 should result in co-localization of DNA-BD and AD, leading to transcription of the SEAP reporter gene from pG5SEAP (Fig. 2a). We used pMUL83, pVP-AIM2, and pG5SEAP to co-transfect HEK293T cells, henceforth referred to as pM-UL83/pVP-AIM2. Several experimental controls were also prepared (Table 2). pM3-VP16 is a strong positive control expressing a fusion of GAL4 DNA-BD to the VP16 AD; pM-53 expresses a fusion of GAL4 DNA-BD to the mouse p53 protein; and pVP16-T expresses a fusion of VP16 AD to the SV40 large T-antigen, which is known to interact with p53 protein. pVP16-CP expresses a fusion of the VP16 AD to a viral coat protein, which does not interact with $\mathrm{p} 53$. Co-transfection of pM-53 and pVP16-T was used as a weak positive control, while co-transfection of pM-53 and pVP16-CP was negative control. Culture supernatants were collected $72 \mathrm{~h}$ post-transfection to assess secreted SEAP levels. As shown in Fig. 2b, pM-UL83/pVP-AIM2 released more SEAP into the culture supernatants than the weak positive control and some other controls $(P<0.01)$, but less SEAP than the strong positive control. This suggested a possible interaction between pUL83 and AIM2. Negative control and auto-activation detection groups produced very low levels of SEAP, indicating that pM and pVP16 had no transcriptional activity by themselves.

We next verified these results by performing coimmunoprecipitation experiments. pM-UL83 and pVPAIM2 were co-transfected into HEK293T cells. Cell lysates were used in immunoprecipitation experiments with anti-AIM2 antibodies, and the antigen-antibody complex was detected by immunoblotting with antipUL83 antibodies. A moderate band at the expected size of recombinant pUL83 (81 kDa) was detected (Fig. 2c). These preliminary analyses support the interaction between pUL83 and AIM2.

\section{pUL83 associates with AIM2 in THP-1 - derived macrophages infected with HCMV}

To determine whether a bona fide interaction between pUL83 and AIM2 occurs in vivo, we further probed this interaction in HCMV-infected cells. THP-1-derived macrophages were mock-infected or infected with the HCMV AD169 strain for $6 \mathrm{~h}, 12 \mathrm{~h}$, and $24 \mathrm{~h}$. The identical cells transfected with poly $(\mathrm{dA}: \mathrm{dT})$ were used as a positive control for AIM2 or a negative control for pUL83. Cell lysates were analyzed by immunoblotting and immunoprecipitation. pUL83 was detected in whole cell lysates at all of the indicated times. Expression of AIM2 was prominent $6 \mathrm{~h}$ and $12 \mathrm{~h}$ post infection, but it was only weakly apparent $24 \mathrm{~h}$ post infection, comparable with the mock-infected control. THP-1-derived cells transfected with poly(dA:dT) expressed AIM2 but not pUL83 (Fig. 3a, WCL fraction). Following immunoprecipitation with the anti-AIM2 antibody, cell lysates were also assayed by immunoblotting. A moderate pUL83-specific band was observed 6 h post infection, 


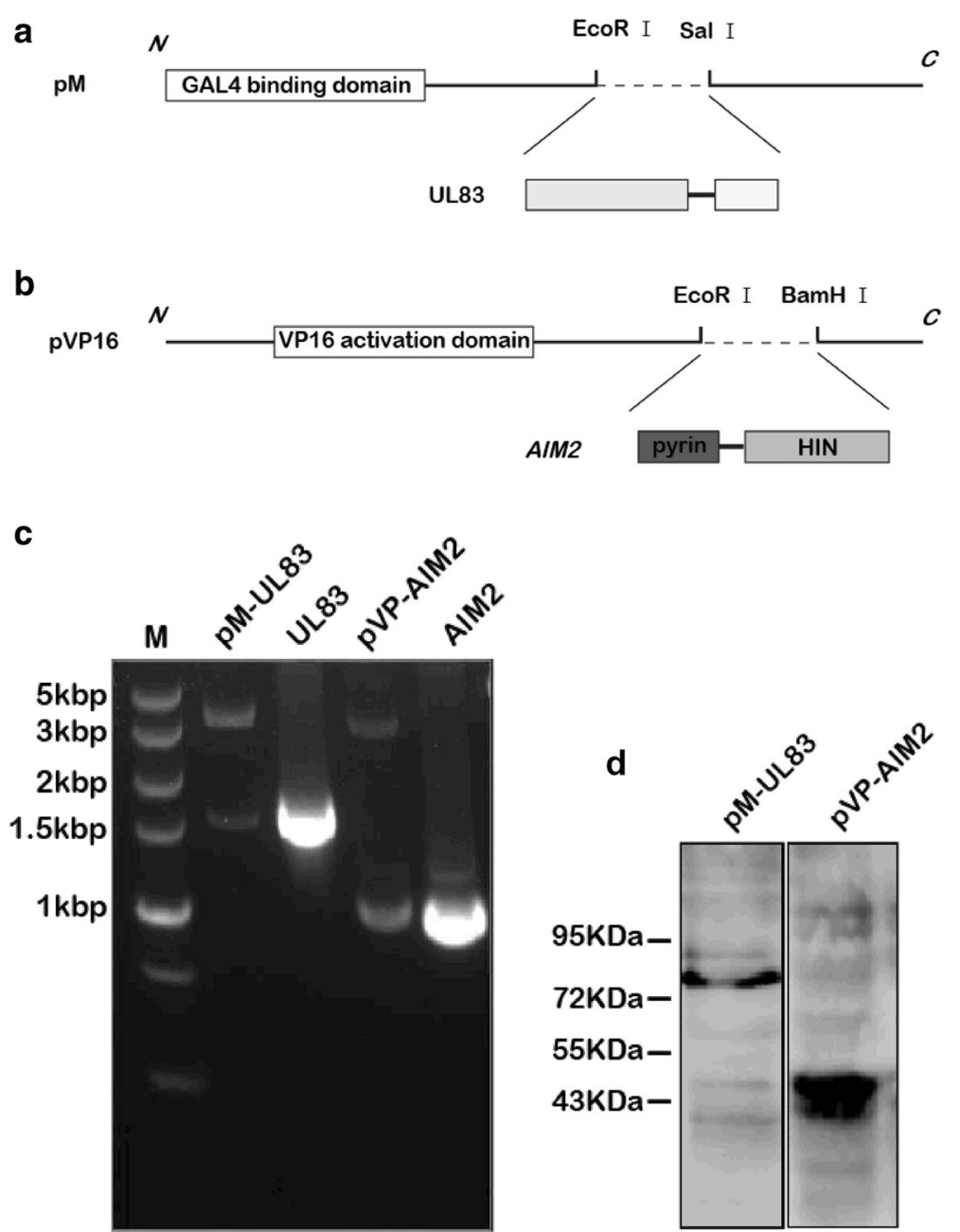

Fig. 1 Construction and expression of recombinant UL83 and AIM2 proteins. a UL83 ORF (1686 bp) was cloned into the MCS of the pM vector for the expression of a fusion of a bait protein (pUL83 herein) with Gal4 DNA BD (147 aa). b AIM2 ORF (1024 bp) was inserted into pVP16 vector to express recombinant AIM2 (the prey protein) fused to VP16 AD (45 aa). c Plasmids digested by EcoRI and Sall or EcoRI and BamHI and PCR products were analyzed by agarose gel electrophoresis. The sizes of the plasmid digestion and PCR products were as anticipated. $\mathbf{d}$ Verified recombinants were used to transfect HEK293T cells for $72 \mathrm{~h}$. The expression of target proteins was assessed by SDS-PAGE with specific antibodies against pUL83 and AIM2. The expected recombinant protein sizes were 81 kDa (BD-pUL83) and 44 kDa (AD-AIM2)

and a more distinct band was seen $12 \mathrm{~h}$ post infection (Fig. 3a, IP: AIM2 fraction). This indicated that pUL83 interacts with AIM2 $6 \mathrm{~h}$ and $12 \mathrm{~h}$ post infection.

We verified these results by performing immunofluorescence experiments. THP-1-derived macrophages were infected with the HCMV AD169 strain for the indicated time periods. We observed that pUL83 (green signal) and AIM2 (red signal) co-localized in the cytoplasm at $6 \mathrm{~h}$ and $12 \mathrm{~h}$ post infection (Fig. 3b, c, white arrow), whereas the AIM2 signal weakened at $24 \mathrm{~h}$ post infection (Fig. 3d). This was consistent with the results of immunoblotting and immunoprecipitation experiments. In summary, our data suggest that pUL83 interacts with AIM2 in HCMV-infected macrophages at the early infection stage.
pUL83/AIM2 complex results in declining abundance of inflammasome proteins

Because the AIM2 inflammasome plays an important role in the host defense against infection, we investigated whether the association between pUL83 and AIM2 affects the subsequent assembly of the inflammasome and IL- $1 \beta$ activation. ASC, pro-caspase- 1 , and pro-IL- $1 \beta$ expression vectors were constructed and used with $\mathrm{pVP}$ AIM2 to co-transfect HEK293T cells. The transient transfectants were named rHEK293T. Poly(dA:dT) was used to transfect rHEK293T to stimulate the activation of the AIM2 inflammasome. To study the impact of pUL83 on the activation of the AIM2 inflammasome, rHEK293T cells were transfected with the pUL83 expression vector prior to poly(dA:dT) stimulation. pUL83 and AIM2 inflammasome-associated proteins 
a

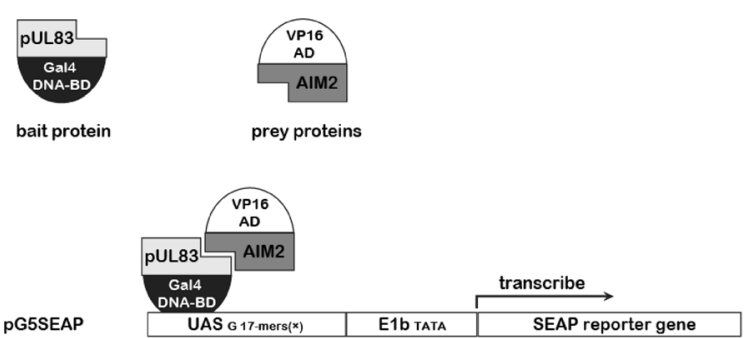

b

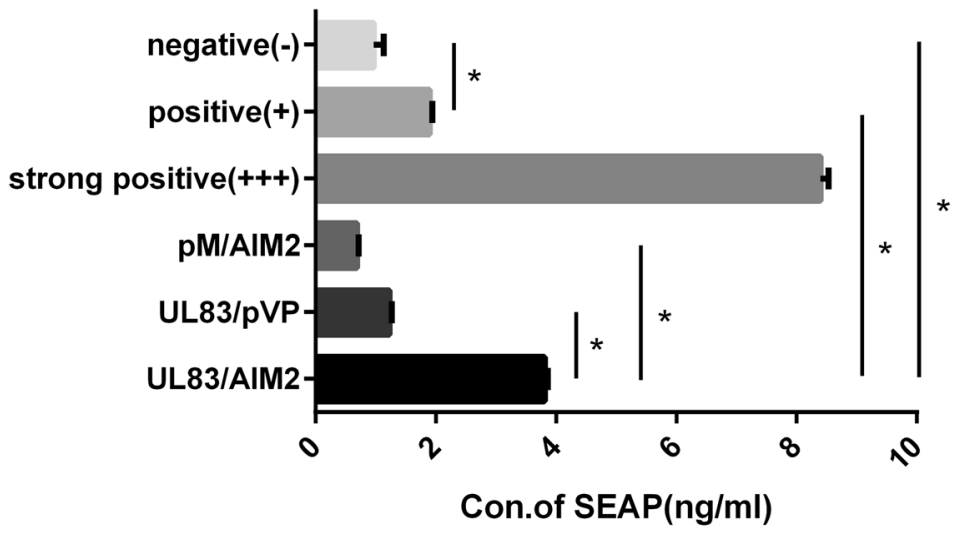

C

WCL IP: AIM2 IgG

WB: pM-pUL83



WB: pVP-AIM2

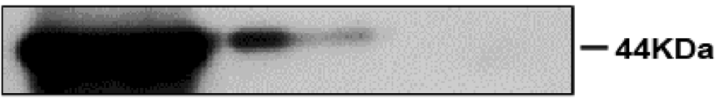

Fig. 2 Detection of the interaction between pUL83 and AIM2. a Schematic diagram of a two-hybrid experiment, adapted from the Matchmaker Mammalian Assay Kit. b Plasmids encoding recombinant pUL83 and AIM2 proteins were used together with pG5SEAP to cO-transfect HEK293T cells for $72 \mathrm{~h}$. Supernatants were then collected and SEAP levels were detected by chemiluminescence at $405 \mathrm{~nm}$. The experiment was repeated three times. Statistical data were analyzed using the $t$-test. c Plasmids encoding recombinant pUL83 and AIM2 were used to transfect HEK293T cells for $72 \mathrm{~h}$. Cells were harvested and lysed with protein lysis buffer, and whole cell lysates were immunoblotted using specific antibodies against pUL83 and AIM2, or immunoprecipitated with the anti-AIM2 antibody and then detected using the anti-pUL83 antibody. IgG was used as a negative control. Data from one representative experiment out of three are presented as the mean $\pm S D$. ${ }^{*} P<0.01$. WCL: whole cell lysates

Table 2 Experimental and control groups

\begin{tabular}{ll}
\hline Experimental & pM-UL83/pVP-AIM2 \\
Bait protein control $^{\Delta}$ & pM-UL83/pVP16 \\
Prey protein control $^{\Delta}$ & pM/pVP-AIM2 \\
Strong positive control $^{\text {Weak positive control }}$ & pM3-VP16 \\
Negative control $^{\Delta}$ & pM-53/pVP16-T \\
Basal control & pM-53/pVP16-CP \\
Untransfected control $^{\Delta}$ & pM/pVP16 \\
\hline
\end{tabular}

$\Delta:$ These controls aim at excluding the possibility of non-carrier self-activation,

$\Delta$ : This control provides the basal expression level of SEAP. ${ }^{\text {a. }}$ This control reveals the background SEAP signal were assayed by immunoblotting. AIM2, ASC, procaspase- 1 , and pro-IL-1 $\beta$ were highly expressed in rHEK293T cells (Fig. 4a, b, line 2). Furthermore, the expression of AIM2, pro-caspase-1, and pro-IL-1 $\beta$ increased both $6 \mathrm{~h}$ and $24 \mathrm{~h}$ after stimulation with poly $(\mathrm{dA}: \mathrm{dT})$. The activated form of caspase-1, p10, and cleaved IL-1 $\beta$ were also detected (Fig. $4 \mathrm{a}, \mathrm{b}$, line 3,4 ), implying that the AIM2 inflammasome was activated. In contrast, pUL83-expressing rHEK293T cells seemed unresponsive to poly ( $\mathrm{dA}: \mathrm{dT}$ ) because the expression of AIM2, pro-caspase-1, and pro-IL1 $\beta$ was reduced, with that of p10 slightly reduced and that of IL-1 $\beta$ dramatically reduced (Fig. 4a, b, line 5, 6) upon poly(dA:dT) stimulation. 




C



$12 \mathrm{~h}$ b

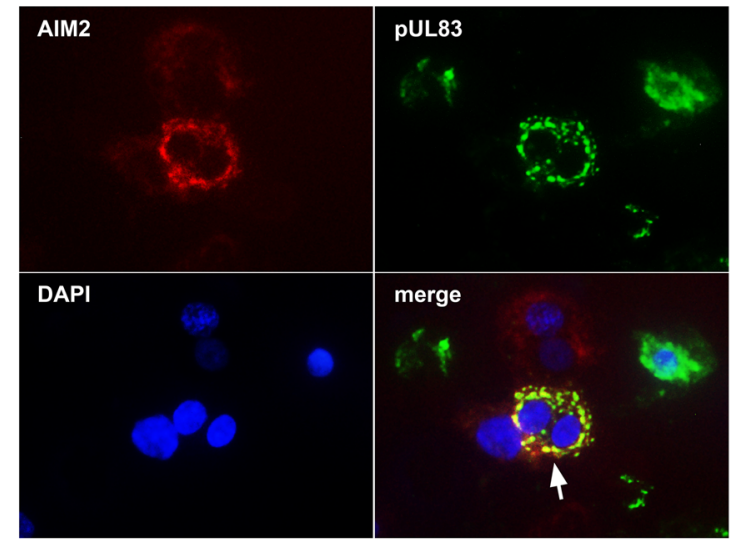

$6 h$

d

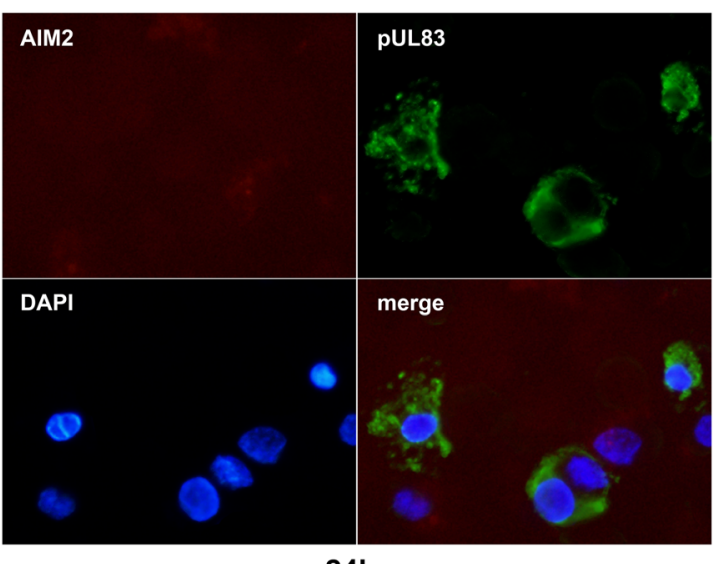

24h

Fig. 3 Detection of the pUL83/AIM2 interaction in HCMV-infected cells. a THP-1 cells were stimulated with PMA (100 $\mathrm{ng} / \mathrm{mL}$ ) to induce cellular differentiation. They were then mock-infected or infected with the HCMV AD169 strain for 6 h, 12 h, or 24 h, or transfected with poly(dA:dT). The cells were harvested and lysed and whole-cell lysates were immunoblotted using specific antibodies against pUL83 and AIM2, or immunoprecipitated with the anti-AIM2 antibody and then detected with anti-pUL83 and anti-AIM2 antibodies. b-d The infected cells were washed and fixed at the indicated time points. Specific antibodies against pUL83 and AIM2 were added and then conjugated with fluorescently tagged secondary antibodies. Cell nuclei were stained with DAPI. P: poly(dA:dT). WCL: whole cell lysates. Red, AIM2; green, pUL83; DAPI (blue), nuclei

To determine whether the reduction of protein levels was caused by the interaction of pUL83 and AIM2 or pUL83 alone, we retested the above experiments in recombinant HEK293T cells expressing ASC, pro-caspase-1, and proIL1 $\beta$, but no AIM2 (Fig. 4a, b, line 7-9). The protein abundance of ASC, pro-caspase-1, and pro-IL-1 $\beta$ was not altered by poly(dA:dT) or pUL83 in AIM2-deficient recombinant HEK293T cells. Additionally, no cleaved caspase- 1 or mature IL-1 $\beta$ was detected. These results collectively indicated that the pUL83/AIM2 complex mediated the attenuation of AIM2 inflammasome proteins and subsequently reduced the cleavage of caspase- 1 and maturation of IL-1 $\beta$.

\section{Discussion}

pUL83 is the most abundant tegument protein and is involved in various biochemical processes in infected cells
[27]. Its ability to engineer immune escape is especially worth noting. Interestingly, even though pUL83 is dispensable for viral growth in human fibroblasts [28], the proliferation of an HCMV variant lacking pUL83 is seriously compromised in monocyte-derived macrophages [27] that constitutively express AIM2 [29]. The different requirements for pUL83 may reflect distinct responses of different cell lines to HCMV. In our previous study involving THP-1 - derived macrophages, we observed an increase in AIM2 levels at the early stage of HCMV infection; however, $24 \mathrm{~h}$ post infection, they returned to the basal level (unpublished data). We proposed that such attenuation of AIM2 inflammasome $24 \mathrm{~h}$ post HCMV infection was linked to pUL83. To investigate the relationship between pUL83 and AIM2 in detail, we performed a two-hybrid assay to assess their putative 


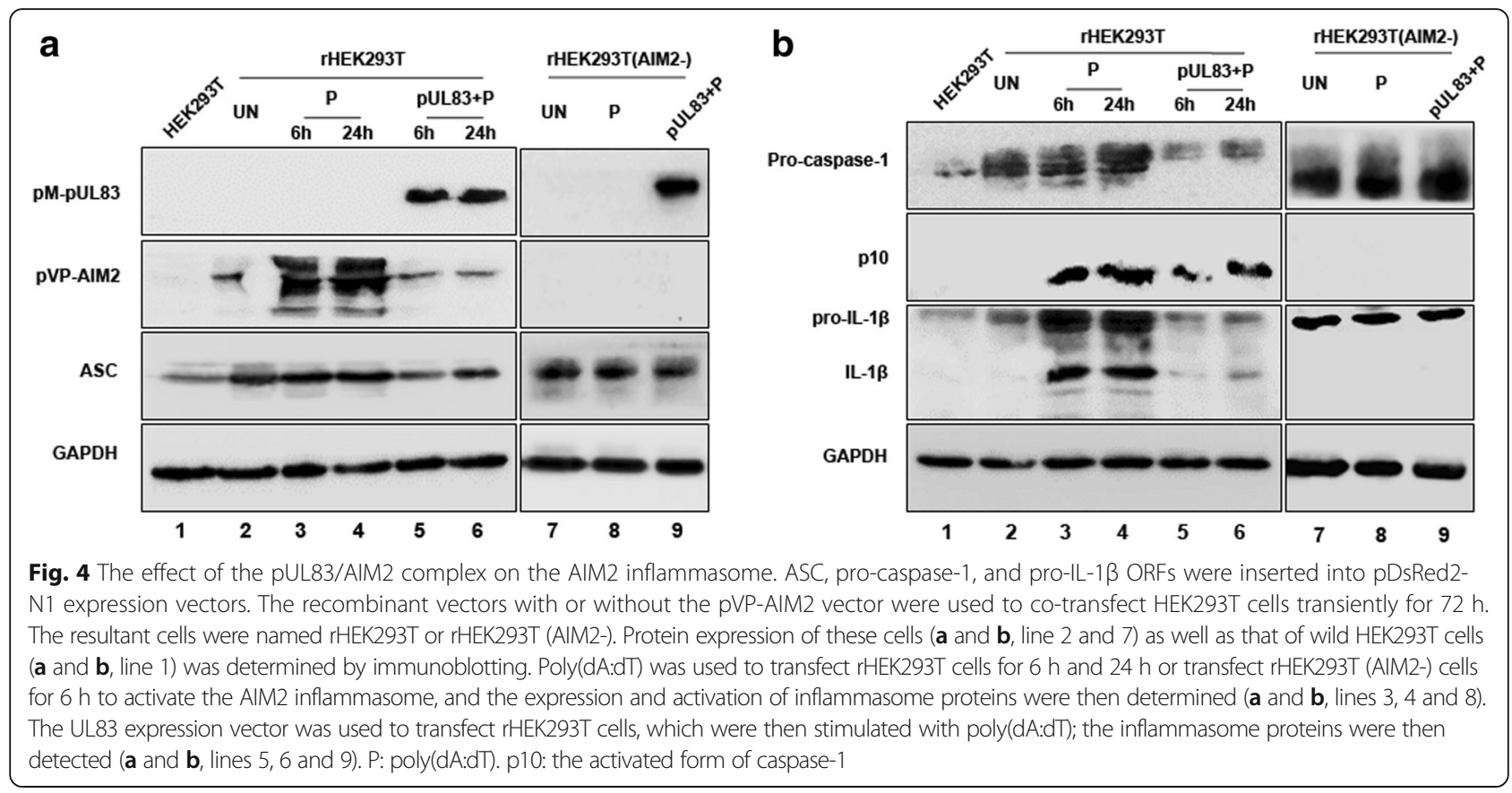

protein-protein interaction in vitro. We successfully constructed pUL83 and AIM2 expression vectors, where the respective proteins were fused with GAL4 BD and AD. These vectors were then used to transfect HEK293T cells. As anticipated, we detected the expression of the reporter gene. This result was confirmed in HCMVinfected THP-1-derived macrophages. We observed that the pUL83/AIM2 complex was indeed formed and localized in the cytoplasm during the early stage of infection, particularly $12 \mathrm{~h}$ post infection, but not $24 \mathrm{~h}$ post infection. In addition, AIM2 protein levels decreased $24 \mathrm{~h}$ post infection, which reinforced our unpublished preliminary observations. Because the AIM2 inflammasome plays an important role in the immune response, we wondered whether the pUL83/AIM2 interaction constitutes one of the immune evasion strategies developed by HCMV, i.e., suppression of the function of the AIM2 inflammasome. Using a published approach [30], we constructed recombinant HEK293T (rHEK293T) cells expressing AIM2, ASC, pro-caspase-1, and pro-IL1 $\beta$, and examined the effect of pUL83 on the AIM2 inflammasome in these cells. Compared with poly(dA:dT)stimulated rHEK293T cells, where a high level of inflammasome activation was maintained, AIM2 protein level, as well as pro-caspase- 1 and pro-IL- $1 \beta$ protein levels, were dramatically reduced when pUL83 was expressed, with a slight decrease in ASC protein level. We next ruled out the independent effect of pUL83 on the indicated proteins (except AIM2). We therefore conclude that the pUL83/AIM2 interaction is responsible for the attenuation of AIM2 inflammasome proteins, followed by reduced cleavage of caspase- 1 and IL- $1 \beta$.
Because these proteins are constitutively expressed in rHEK293T cells, we surmise that the decline in protein abundance results from increased protein degradation rather than reduced gene expression. According to our results, caspase- 1 activated form, p10, and IL- $1 \beta$ were still weakly detected in rHEK293T cells in the presence of pUL83, indicating that rather than preventing the assembly of the AIM2 inflammasome, pUL83 facilitates the degradation of the assembled AIM2 inflammasome through binding to AIM2. Autophagy is a cell homeostatic process that mediates the degradation of cytosolic protein aggregates [31]. An increasing number of studies has shown involvement of autophagy in the regulation of the inflammasome [32, 33]. For instance, autophagy controls the production of IL-1 $\beta$ through sequestering and targeting of pro-IL-1 $\beta$ for lysosomal degradation [34]. In addition, autophagocytosis-deficient Atg16-/mice accumulated excessive IL- $1 \beta$ in response to LPS [35]. Recently, Nurmi et al. found that intraperitoneal administration of a hemin derivative depleted ASC in mice macrophages, which was attributed to the autophagy pathway [36]. Moreover, a previous study showed that the AIM2 inflammasome could trigger and in turn be degraded by autophagy [37]. We therefore propose that the pUL83/AIM2 complex might enhance the autophagy pathway and accelerate the degradation of inflammasome proteins.

\section{Conclusion}

In summary, our data indicate that the HCMV tegument protein pUL83 binds to cellular AIM2, which partially contributes to the attenuation of the AIM2 inflammasome 
proteins $24 \mathrm{~h}$ post $\mathrm{HCMV}$ infection and reduced activation of caspase- 1 and IL-1 $\beta$. This effect of the pUL83/ AIM2 interaction may facilitate the latency of HCMV, hence informing the treatment of latent HCMV infections. However, our data are based on in vitro models of infection and should be verified in further experiments. The proposed biological significance of the pUL83/AIM2 interaction should be investigated in depth in an in vivo infection model, by either overexpressing or deleting the UL83 gene. Other experimental approaches such as fluorescence resonance energy transfer (FRET) and highresolution electron microscopy should be used to obtain physical evidence of this interaction.

\begin{abstract}
Abbreviations
AD: Activating domain; AGE: Agarose gel electrophoresis; AIM2: Absent in melanoma 2; ASC: Apoptosis-associated speck-like; BD: Biding domain; DAPI: 4',6-diamidino-2-phenylindole; dsDNA: Double-stranded DNA; HCMV: Human cytomegalovirus; HIN: Hematopoietic IFN-inducible nuclear; IE: Immediate-early; IFN: Interferon; IL: Interleukin; IRF1: Interferon regulatory factor 1; MCMV: Mouse cytomegalovirus; MCS: Multiple cloning site; MIEP: Major immediate early promoter; NK: Natural killer; ORF: Open reading frame; PBS: Phosphate buffer saline; PMA: Phorbol myristate acetate; RT-PCR: Reversetranscription polymerase chain reaction; SDS-PAGE: Sodium dodecyl sulfate polyacrylamide gel electrophoresis; SEAP: Secreted embryonic alkaline phosphatase; UAS: Upstream activating sequence; ZBP1: Z-DNA binding protein 1
\end{abstract}

\section{Acknowledgements}

The HCMV AD169 laboratory strain was a kind present of Wuhan Institute of Virology, Chinese Academy of Sciences. We would like to thank Editage [http://www.editage.cn/] for English language editing.

\section{Funding}

This research is supported by the National Natural Science Foundation of China (Grant No. 81271807, 81301425).

\section{Availability of data and materials}

All data generated or analyzed during this study are included in this published article.

\section{Authors' contributions}

HY designed the study, constructed the recombinants, performed the twohybrid and immunofluorescence assay, operated the statistical analyses, and drafted the manuscript. DM, HY-H, YL, and YY-L helped to construct the recombinants and assays. $L L-L$ and $L X-L$ helped to design the study and modify the manuscript. FF coordinated and designed the study and modified the manuscript. All authors read and approved the final manuscript.

\section{Competing interests}

The authors declare that they have no competing interests.

\section{Consent for publication}

Not applicable.

Ethics approval and consent to participate

Not applicable.

Received: 2 July 2016 Accepted: 18 December 2016 Published online: 20 February 2017

\section{References}

1. Marshall EE, Geballe AP. Multifaceted evasion of the interferon response by cytomegalovirus. J Interf Cytok Res. 2009;29:609-19.

2. Lucin P, Mahmutefendic H, Blagojevic Zagorac G, llic TM. Cytomegalovirus immune evasion by perturbation of endosomal trafficking. Cell Mol Immunol. 2015;12:154-69.
3. Lu A, Magupalli VG, Ruan J, Yin Q, Atianand MK, Vos MR, et al. Unified polymerization mechanism for the assembly of ASC-dependent inflammasomes. Cell. 2014;156:1193-206.

4. Landolfo S, Gariglio M, Gribaudo G, Lembo D. The human cytomegalovirus. Pharmacol Ther. 2003;98:269-97.

5. Varnum SM, Streblow DN, Monroe ME, Smith P, Auberry KJ, Pasa-Tolic L, et al. Identification of proteins in human cytomegalovirus (HCMV) particles: the HCMV proteome. J Virol. 2004;78:10960-6.

6. Somogyi T, Michelson S, Masse MJ. Genomic location of a human cytomegalovirus protein with protein kinase activity (PK68). Virology. 1990;174:276-85.

7. Cristea IM, Moorman NJ, Terhune SS, Cuevas CD, O'Keefe ES, Rout MP, et al. Human cytomegalovirus pUL83 stimulates activity of the viral immediate-early promoter through its interaction with the cellular IFI16 protein. J Virol. 2010;84:7803-14.

8. Gilbert MJ, Riddell SR, Plachter B, Greenberg PD. Cytomegalovirus selectively blocks antigen processing and presentation of its immediate-early gene product. Nature. 1996;383:720-2.

9. Odeberg J, Plachter B, Branden L, Soderberg-Naucler C. Human cytomegalovirus protein pp 65 mediates accumulation of HLA-DR in lysosomes and destruction of the HLA-DR alpha-chain. Blood. 2003;101:4870-7.

10. Browne EP, Shenk T. Human cytomegalovirus UL83-coded pp 65 virion protein inhibits antiviral gene expression in infected cells. Proc Natl Acad Sci U S A. 2003;100:11439-44.

11. Arnon TI, Achdout H, Levi O, Markel G, Saleh N, Katz G, et al. Inhibition of the NKp30 activating receptor by pp 65 of human cytomegalovirus. Nat Immunol. 2005;6:515-23.

12. Schattgen SA, Fitzgerald KA. The PYHIN protein family as mediators of host defenses. Immunol Rev. 2011;243:109-18.

13. Fernandes-Alnemri T, Yu JW, Datta P, Wu JH, Alnemri ES. AlM2 activates the inflammasome and cell death in response to cytoplasmic DNA. Nature. 2009;458:509-U5.

14. Hornung V, Ablasser A, Charrel-Dennis M, Bauernfeind F, Horvath G, Caffrey DR, et al. AIM2 recognizes cytosolic dsDNA and forms a caspase-1-activating inflammasome with ASC. Nature. 2009;458:514-8.

15. Burckstummer T, Baumann C, Bluml S, Dixit E, Durnberger $G$, Jahn $H$, et al. An orthogonal proteomic-genomic screen identifies AIM2 as a cytoplasmic DNA sensor for the inflammasome. Nat Immunol. 2009;10:266-72.

16. Lu A, Kabaleeswaran V, Fu TM, Magupalli VG, Wu H. Crystal structure of the F27G AIM2 PYD mutant and similarities of its self-association to DED/DED interactions. J Mol Biol. 2014;426:1420-7.

17. Morrone SR, Matyszewski M, Yu X, Delannoy M, Egelman EH, Sohn J. Assembly-driven activation of the AIM2 foreign-dsDNA sensor provides a polymerization template for downstream ASC. Nat Commun. 2015:6:7827.

18. Rathinam VAK, Jiang ZZ, Waggoner SN, Sharma S, Cole LE, Waggoner L, et al. The AIM2 inflammasome is essential for host defense against cytosolic bacteria and DNA viruses. Nat Immunol. 2010;11:395-403.

19. Jin TC, Perry A, Jiang JS, Smith P, Curry JA, Unterholzner L, et al. Structures of the HIN domain: DNA complexes reveal ligand binding and activation mechanisms of the AIM2 inflammasome and IFI16 receptor. Immunity. 2012;36:561-71

20. Botero JE, Contreras A, Parra B. Profiling of inflammatory cytokines produced by gingival fibroblasts after human cytomegalovirus infection. Oral Microbiol Immunol. 2008;23:291-8.

21. van de Berg PJ, Heutinck KM, Raabe R, Minnee RC, Young SL, van Donselaar-van der Pant KA, et al. Human cytomegalovirus induces systemic immune activation characterized by a type 1 cytokine signature. J Infect Dis. 2010;202:690-9.

22. DeFilippis VR, Alvarado D, Sali T, Rothenburg S, Fruh K. Human cytomegalovirus induces the interferon response via the DNA sensor ZBP1. J Virol. 2010;84:585-98.

23. Li T, Chen J, Cristea IM. Human cytomegalovirus tegument protein pUL83 inhibits IFI16-mediated DNA sensing for immune evasion. Cell Host Microbe. 2013;14:591-9.

24. Depto AS, Stenberg RM. Regulated expression of the human cytomegalovirus pp 65 gene: octamer sequence in the promoter is required for activation by viral gene products. J Virol. 1989;63:1232-8.

25. Aspenstrom P, Olson MF. Yeast two-hybrid system to detect protein-protein interactions with Rho GTPases. Methods Enzymol. 1995;256:228-41.

26. Keegan K, Cooper JA. Use of the two hybrid system to detect the association of the protein-tyrosine-phosphatase, SHPTP2, with another SH2containing protein, Grb7. Oncogene. 1996;12:1537-44. 
27. Chevillotte M, Landwehr S, Linta L, Frascaroli G, Luske A, Buser C, et al. Major tegument protein pp 65 of human cytomegalovirus is required for the incorporation of pUL69 and pUL97 into the virus particle and for viral growth in macrophages. J Virol. 2009;83:2480-90.

28. Schmolke S, Kern HF, Drescher P, Jahn G, Plachter B. The dominant phosphoprotein pp 65 (UL83) of human cytomegalovirus is dispensable for growth in cell culture. J Virol. 1995;69:5959-68.

29. DeYoung KL, Ray ME, Su YA, Anzick SL, Johnstone RW, Trapani JA, et al. Cloning a novel member of the human interferon-inducible gene family associated with control of tumorigenicity in a model of human melanoma. Oncogene. 1997;15:453-7.

30. Seshadri S, Duncan MD, Hart JM, Gavrilin MA, Wewers MD. Pyrin levels in human monocytes and monocyte-derived macrophages regulate IL-1 beta processing and release. J Immunol. 2007;179:1274-81.

31. Svenning S, Johansen T. Selective autophagy. Essays Biochem. 2013:55:79-92.

32. Rathinam VA, Vanaja SK, Fitzgerald KA. Regulation of inflammasome signaling. Nat Immunol. 2012;13:333-42.

33. Saitoh T, Akira S. Regulation of inflammasomes by autophagy. J Allergy Clin Immunol. 2016;138:28-36.

34. Harris J, Hartman M, Roche C, Zeng SJG, O'Shea A, Sharp FA, et al. Autophagy controls IL-1 beta secretion by targeting Pro-lL-1 beta for degradation. J Biol Chem. 2011;286:9587-97.

35. Saitoh T, Fujita N, Jang MH, Uematsu S, Yang BG, Satoh T, et al. Loss of the autophagy protein Atg16L1 enhances endotoxin-induced IL-1 beta production. Nature. 2008:456:264-U68.

36. Nurmi K, Kareinen I, Virkanen J, Rajamaki K, Kouri VP, Vaali K, et al. Hemin and Cobalt Protoporphyrin Inhibit NLRP3 Inflammasome Activation by Enhancing Autophagy: A Novel Mechanism of Inflammasome Regulation. J Innate Immun. 2017;9(1):65-82.

37. Shi CS, Shenderov K, Huang NN, Kabat J, Abu-Asab M, Fitzgerald KA, et al. Activation of autophagy by inflammatory signals limits IL-1 beta production by targeting ubiquitinated inflammasomes for destruction. Nat Immunol. 2012:13:255-63.

\section{Submit your next manuscript to BioMed Central and we will help you at every step:}

- We accept pre-submission inquiries

- Our selector tool helps you to find the most relevant journal

- We provide round the clock customer support

- Convenient online submission

- Thorough peer review

- Inclusion in PubMed and all major indexing services

- Maximum visibility for your research

Submit your manuscript at www.biomedcentral.com/submit

) Biomed Central 